Public Abstract

First Name:Yajun

Middle Name:

Last Name:Liu

Adviser's First Name:Dongchu

Adviser's Last Name:Sun

Co-Adviser's First Name:

Co-Adviser's Last Name:

Graduation Term:SS 2012

Department:Statistics

Degree:PhD

\title{
Title:BAYESIAN ANALYSIS OF SPATIAL AND SURVIVAL MODELS WITH APPLICATIONS OF COMPUTATION TECHNIQUES
}

This dissertation discusses the methodologies of applying Bayesian hierarchical models to different data with geographical characteristics or with survival time. Spatial effects are captured by a conditional autoregressive (CAR) model. Several survival models with spatial effect are also discussed to analyze the survival pattern of colorectal cancer based on geographical factors and patients' disease conditions.

A couple of advanced sampling techniques are applied to improve convergence, or sample from irregular posterior distributions. The overall performance has improved by using an algorithm on regular Gibbs sampling. In addition, spatial confounding issue arising from linear mixed models is discussed. Different estimators are compared for their mean squared errors and variances.

The spatial analysis with survival models provides statisticians new perspectives for cancer survivors analysis. The methods of sampling help to solve difficult sampling problems from non-standard densities. Finally, the spatial confounding discussion will help researchers find better estimators balanced between small bias and variances. 